\title{
3-D Shape Matching for Face Analysis and Recognition
}

\author{
Wei Quan, Bogdan J. Matuszewski and Lik-Kwan Shark \\ Robotics and Computer Vision Research Laboratory, Applied Digital Signal and Image Processing (ADSIP) Research \\ Centre, University of Central Lancashire, Preston PR1 2HE, U.K.
}

\begin{abstract}
Keywords: Face Recognition, Shape Matching and Modelling, Isometric Embedding Representation, Non-Rigid
\end{abstract} Deformation Registration.

\begin{abstract}
The aims of this paper are to introduce a 3-D shape matching scheme for automatic face recognition and to demonstrate its invariance to pose and facial expressions. The core of this scheme lies on the combination of non-rigid deformation registration and statistical shape modelling. While the former matches 3-D faces regardless of facial expression variations, the latter provides a low-dimensional feature vector that describes the deformation after the shape matching process, thereby enabling robust identification of 3-D faces. In order to assist establishment of accurate dense point correspondences, an isometric embedding shape representation is introduced, which is able to transform 3-D faces to a canonical form that retains the intrinsic geometric structure and achieve shape alignment of 3-D faces independent from individual's facial expression. The feasibility and effectiveness of the proposed method was investigated using standard publicly available Gavab and BU-3DFE databases, which contain faces expressions and pose variations. The performance of the system was compared with the existing benchmark approaches and it demonstrates that the proposed scheme provides a competitive solution for the face recognition task with real-world practicality.
\end{abstract}

\section{INTRODUCTION}

Human face is one of the widely used biometric features. It is used for subject identification with advantages, including: naturalness, non-contact and non-intrusiveness. Face recognition and related research have been for many years of great interest to computer vision and image processing communities, which has been exploited for applications such as public security (Chellappa, 1995), fraud prevention (Jafri, 2009) and crime prevention and detection (Kong, 2005). A fair amount of efforts have been made on the development of 2-D face recognition systems using intensity images as input data in the past. Despite 2D face recognition systems have a capability to achieve good performance under controlled conditions, they are still facing great difficulties as facial appearances can vary significantly even for the same individual due to differences in pose, lighting conditions and expressions ( $\mathrm{Lu}, 2003)$.

By using 3-D face information, it is expected to overcome the challenges faced by 2-D face recognition systems and improve the performance of face recognition, since the 3-D faces contain explicit
3-D geometry which can be used to handle the variations of face pose and expressions ( $\mathrm{Lu}, 2008)$. Unlike simple image normalisation and alignment in 2-D face recognition, 3-D systems usually require more sophisticated shape matching methodologies in order to standardise data prior to feature extraction. This is a major challenge in 3-D face recognition as facial shapes of the same person can be very different due to diverse expressions (Jenkins, 2011). Face recognition based on range images has been investigated by a number of researchers in order to simplify 3-D face recognition and avoid the complicated shape matching process (Pan, 2003), (Chang, 2003; Huang, 2012). This type of method does not use all the 3-D information available from a face and often ignores the shape deformation caused by the facial expression. Chua et al. (Chua, 2002) introduced a modified version of Point Signature to recognize 3-D face scans with facial expressions. A method based on the integration of matching results from multiple regions around the nose was presented by Chang et al. (Chang, 2006). Bronstein et al. (Bronstein, 2005) introduced an isometric model of facial surfaces in order to derive an expressioninvariant face representation for 3-D face 


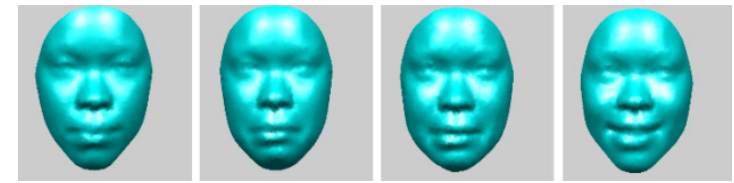

(a)



(b)

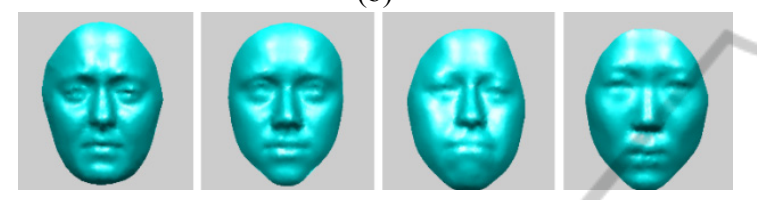

(c)



(d)

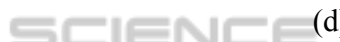

Figure 1: Examples of isometric embedding transformation: (a) original faces from the same subject across facial expressions; (b) transformed faces from row (a); (c) original faces from different subjects; (d) transformed faces from row (c).

recognition. The method relies on the assumption that face is approximately isometric and the geodesic distances among the points on the facial surface are preserved under facial expressions. $\mathrm{Lu}$ et al. ( $\mathrm{Lu}$, 2008) proposed a 3-D deformation modelling scheme that is capable of handling facial expression variations. With assistance of automatically extracted landmarks, a deformable model was built linearly, and the method has been applied to the problem of matching multiview facial scans to a neutral facial model. Mpiperis et al. (Mpiperis, 2007) proposed a geodesic polar parameterisation of the facial surface. With the parameterisation, the intrinsic surface attributes do not change under the isometric deformation caused by facial expressions. A representation of patch geodesic moment for 3-D face recognition was proposed by Hajati (Hajati, 2012). This patch-based local pattern combines local and global geodesic paths with patches in a coarse to fine hierarchical computation for each surface point. One of the common approaches for 3-D face recognition is to register a probe face to each gallery face and then calculate the sum of the distances between their points. This is computationally expensive and sensitive to facial expresion variations. To improve the efficiency,
Mohammadzade et al. (Mohammadzade, 2013) introduced an iterative normal point method for finding the corresponding points between a probe face and gallery faces. The method can also cope with the changes of facial expressions.

The statistical-model based face recognition has been one of the most successful techniques over the past few decades. In this paper, a 3-D shape matching scheme is proposed, which works in conjunction with statistical shape modelling to complete the task of face recognition. An isometric embedding shape representation is used for the neutralisation of 3-D faces with facial expressions so that the non-rigid deformation problem caused by facial expressions can be handled and the locality preserving projection (LPP) is used in the dimensionality reduction process to construct the statisitical shape model. One of the novel contribution of the paper is the use of the isometric embedding transformation. It not only enables the shape representation of 3-D faces indepdenet from individual's facial expressions, but also reduces the complexity of the dense point correspondence search in the shape matching. Further more, the proposed methods uses only 3-D point clouds with texture not being used at all. The method is therefore inherently invariant to variations in scenes illumination conditions, background clutter and to some extent angle of view. This is in striking contrast to the method based on texture where these factors severely limit their practical applicability.

The remainder of the paper is organised as follows. Section 2 introduces the concept of the isometric embedding shape representation. Then, further shape alignment based on thin-plate spline (TPS) surface warping is described in Section 3. Section 4 explains the LLP dimensionality reduction method. Section 5 describes the fitting approach for new dataset. The experimental results are shown in Section 6 with concluding remarks in Section 7.

\section{ISOMETRIC EMBEDDING SHAPE REPRESENTATION}

Expression-invariance is of particular importance as most of the face recognition systems have to cope with various facial expressions. Isometric embedding shape representation was proposed by Elad and Kimmel (Elad, 2003), which provides a possibility for expression-invariant face recognition. In this work, the isometric embedding shape representation is used to minimise the shape 


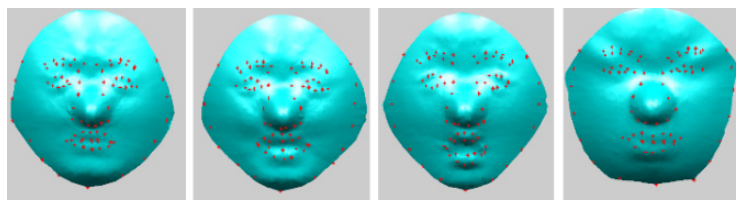

(a)

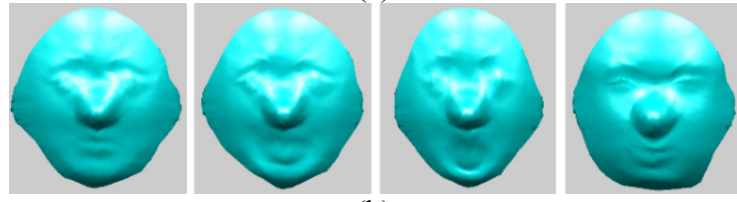

(b)

Figure 2: Further shape alignment: (a) canonical faces with 83 facial landmarks; (b) reference faces after alignment.

variation across different 3-D faces from the same individual, with the shape variations mainly caused by facial expressions. Isometric embedding shape representation assumes that different 3-D facial scans from the same individual are isometric to his/her 3-D facial scan of the neutral expression, therefore they can be transformed to approximate the shape of the neutral face. The new representation provided by the isometric embedding transformation is known as canonical form, which has the Euclidean metric structure of the facial surface with the geodesic replaced by Euclidean distance (Bronstein, 2007).

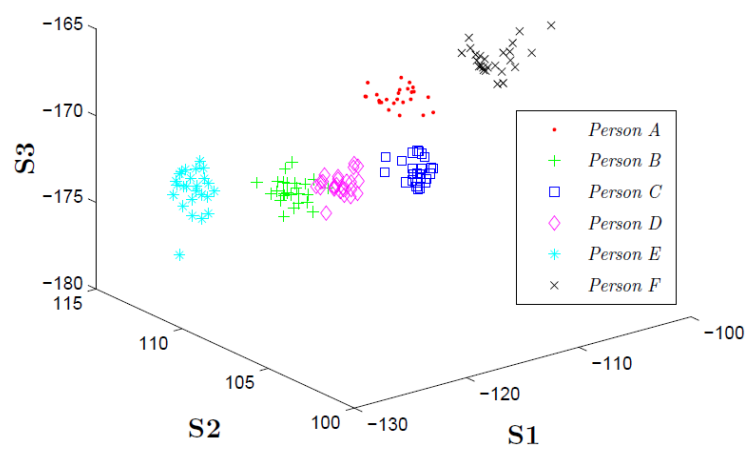

Figure 3: 3-D projection of LPP feature subspace for six subjects with various facial expressions.

Multidimensional Scaling (MDS) is one of the popular approaches for obtaining the canonical form of faces (Bronstein, 2006). Given a smooth surface that has been sampled at $N$ points $\left\{\xi_{1}, \ldots, \xi_{N}\right\}$, (typically, $N$ is approximately 3000 for a reasonable surface description of a human face), with a matrix of geodesic distance $\Delta=\left[\delta_{i j}\right]$ between each pair of surface points, each point $\theta$ in the embedding space $\mathbb{R}^{3}$ is given by $\theta_{k}=\left(\theta_{k}^{1}, \theta_{k}^{2}\right)$. The geodesic distances are calculated by some explicit or efficiently approximated function $d_{s}\left(\xi_{i}, \xi_{j}\right)=\delta_{i j}=d_{\mathbb{R}^{3}}\left(\theta_{i}, \theta_{j}\right)$, where $d_{s}$ represents geodesic distance. MDS solves the following optimisation problem

$$
\Theta=\underset{\theta}{\operatorname{argmin}} \sigma(\Theta, \Delta)
$$

where $\Theta=\left(\theta_{1}, \ldots, \theta_{N}\right)$ is an $N \times 3$ matrix of coordinates in $\mathbb{R}^{3}$ corresponding to points $\left\{\xi_{1}, \ldots, \xi_{N}\right\}$ on the surface, and $\sigma$ is an embedding error criterion function, measuring the discrepancies between the original geodesic distances and the embedding space Euclidean distances. The most common choices include the raw stress

$$
\sigma_{\text {raw }}(\Theta, \Delta)=\sum_{i>j}\left(d_{i j}(\Theta)-\delta_{i j}\right)^{2}
$$

and the normalised stress

$$
\sigma_{\text {norm }}(\Theta, \Delta)=\frac{\sum_{i>j}\left(d_{i j}(\Theta)-\delta_{i j}\right)^{2}}{\sum_{i>j} d_{i j}^{2}(\Theta)}
$$

where $d_{i j}(\Theta)$ is the short notation for $d_{\mathbb{R}^{2}}\left(\theta_{i}, \theta_{j}\right)$. The usual algorithms for solving the MDS problem are Newton, Quasi-Newton and gradient-descent optimisation (Bronstein, 2006). In this work, the Newton optimisation approach is applied to achieve the isometric embedding transformation. Figure 1 shows examples of 3-D faces before and after the transformation. From Figure 1(b), it is seen that the 3 -D shapes of the same subject with different facial expressions appear to be similar after the transformation, and this illustrates the goodness of shape variance offered by the transformation for recognition of a person independent from individual's facial expressions.

\section{FURTHER SHAPE ALIGNMENT}

Alignment of 3-D faces in the training dataset is essential for statistical model construction since inappropriate alignment will result in erroneous dense point correspondences. As the shape deformation related to the facial expression is much reduced by the isometric embedding transformation, the remaining differences is the shape across the faces of different subjects in the training dataset as shown in Figures 1 (c) and (d). A further shape alignment is achieved by Thin-Plate Spline (TPS) surface warping (Quan, 2009). The alignment starts 
with the selection of a reference face from the training dataset. The reference canonical face usually contains a neutral facial expression. It is then warped to align with other canonical faces in the training dataset using the TPS transformation, which is calculated based on the selected landmarks on the faces. For the results reported here, 83 landmarks provided in the BU-3DFE database (Yin, 2006), were used for that purpose. For the Gavab database (Moreno, 2004), the same 83 landmarks were manually labelled on each training face. Figure 2 shows examples of the further shape alignment. It can be seen that after the alignment the transformed canonical reference faces of different subjects in Figure 2(a) are closely matched to each other in Figure 2(b). Thus the pair-wise dense point correspondences between the warped canonical reference face and other canonical faces from the training dataset can be established by pairing points based on the closest distance metric (Quan, 2009). These corresponding points later provide the basis for building a statistical shape model.

\section{DIMENSIONALITY- REDUCTION METHOD}

Dimensionality-reduction is usually applied with statistical shape modelling approaches. Principal component analysis (PCA) and linear discriminant analysis (LDA) are the popular choices, when data lie in the linear subspace of a high dimensional space. The locality preserving projection (LPP) is able to handle a wider range of data variability while preserving local structure, which often links the structure of the manifold $(\mathrm{He}, 2005)$. Therefore it suggests that LPP can perform well on the task of face recognition even under the influence of facial expressions.

LPP is a technique with isometric mapping from the original data space to the reduced low dimensional space (Hastie, 2001). The optimality criterion to minimise,

$$
\frac{1}{2} \sum_{k} \sum_{i, j}\left(y_{i}^{(k)}-y_{j}^{(k)}\right)^{2} s_{i, j}, i, j=1,2, \ldots, N, k=1,2, \ldots, d
$$

where $y_{j}^{(k)}=\mathbf{a}_{k}^{T} \mathbf{x}_{j}$ describes the $k$-th coordinate of vector $\mathbf{y}_{j} \in \mathbb{R}^{d}$ representing the $j$-th training face $\mathbf{x}_{j} \in \mathbb{R}^{n}$ in a lower dimensional space, $d<<n$. $S=\left[s_{i, j}\right]$ is the adjacency matrix, which defines the weighted similarity graph for all connected vertices, and can be computed as,

$$
s_{i, j}=\exp \left(-\left\|x_{i}-x_{j}\right\|^{2} / 2 \delta\right)
$$

where $\delta$ is calculated as,

$$
\delta=\frac{1}{N} \sum_{i=1}^{N} \min _{j x_{i} \neq x_{j}}\left\|\mathbf{x}_{i}-\mathbf{x}_{j}\right\|^{2}
$$

To enforce a non-trivial solution to the problem posed in Equation 4, vectors $\mathbf{a}_{i}$ should fulfil the following constraint, $\mathbf{a}_{i}^{T} X D X^{T} \mathbf{a}_{i}^{T}=1$. In this case, the solution is given by solving the following generalised eigenvalue problem:

$$
X L X^{T} \mathbf{a}_{i}=\lambda_{i} X D X^{T} \mathbf{a}_{i}
$$

where $X$ contains all the training faces, $L=D-S$ with $L=\left[d_{i, j}\right], \quad d_{i, i}=\sum_{j=1}^{N} s_{i, j}, \quad d_{i, i}=0, \quad$ for all $i \neq j, \forall d \in D$.

An example from the separability study of the LPP-based method by reducing the dimensionality for visualisation is provided in Figure 3, where the LPP features of 150 faces from six persons with 25 expressions per person (taken from BU-3DFE database) are projected to a 3-D space. As can be seen, the output samples are well grouped in six clusters corresponding to the six individuals and exhibitings a good inter-person separability against facial expressions even in a low dimensional space.



Figure 4: Rank-1 recognition accuracy versus feature subspace dimensions.

\section{NEW DATASET FITTING}

After the eigenvectors of the constructed models are extracted, the estimation of feature vectors can be 


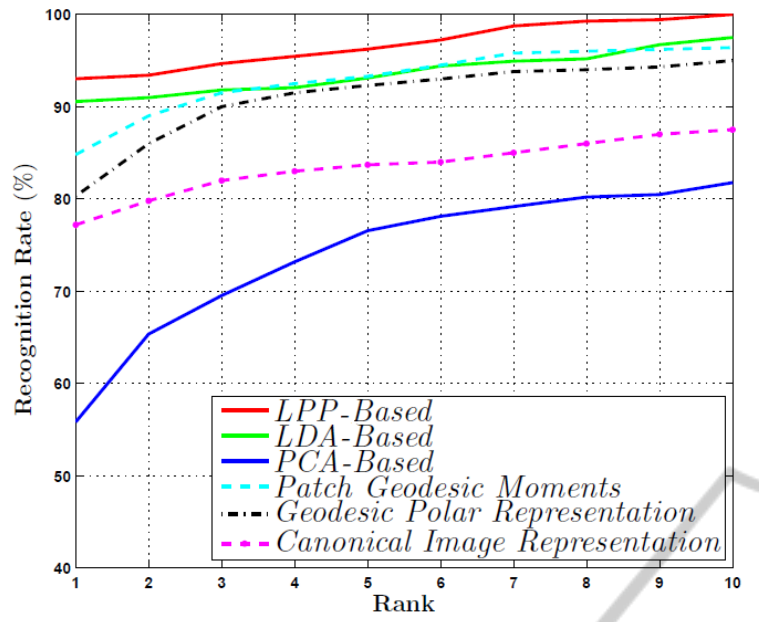

Figure 5: CMC curves of recognition accuracy for the proposed and exiting benchmark methods.

undertaken in order to synthesize faces in a new set of data. This is usually achieved by recursive data registration, such as modified Iterative Closest Point (ICP), in which the shape and pose parameters are iteratively estimated in turn. While pose parameters control the orientation and position of the model, shape parameters encapsulate deformation of the model. In order to solve all unknown parameters effectively, the following standard optimisation scheme is used:

1. Apply isometric embedding transformation to the new face.

2. Align model and new face by using their centroid point, to minimise translation difference.

3. Apply isometric embedding transformation to both model and new face.

4. Use ICP algorithm to estimate dense point correspondences between transformed model and new face.

5. Estimate feature vector $\boldsymbol{\alpha}$ for the new face using back-projection based on the original data, described as

$$
\boldsymbol{\alpha}=A_{o p t}^{T} \hat{\mathbf{x}}
$$

where $\hat{\mathbf{x}}$ is the estimated dense point correspondences for a test face and $A_{o p t}$ is the matrix containing eigenvectors.

6. Generate a new instance of the statistical model using the feature vector and repeat steps 2 to 5 until the pre-set convergence condition is reached.

In this optimisation scheme, the isometric embedding shape representation serves the similar purpose as applied to the training dataset, which reduces partial shape difference on both model and new face. This potentially improves the dense point correspondence search and provides better estimation for the later stages of the optimisation. Since vector $\boldsymbol{\alpha}$ controls the shape of the model, it can be used as the feature vector for classifying face identity.

\section{EXPERIMENTAL RESULTS}

To demonstrate the effectiveness of the proposed method for the purpose of face verification and recognition, two public databases, BU-3DFE (Yin, 2006) and Gavab (Moreno, 2004), were utilised for the evaluation in this work. While the former was used for evaluating performance related to the dimensions of feature subspace and facial expression changes; the latter was used to further observe its robustness to facial expression changes as well as pose variations. For the sake of simplicity, the nearest-neighbour classifier was chosen for the feature classification in this study. The two popular dimensionality-reduction methods, PCA and LDA, are also compared along with other benchmark approaches.

\subsection{Experiments on BU-3DFE Database}

The BU-3DFE database consists of 2,500 3-D faces from 100 subjects, ranging from 18 to 70 years old, with a variety of ethnic origins including White, Black, East-Asian, Middle East Asian, Indian and Hispanic Latino. Each subject has seven expressions, one neutral and six universal expressions: anger, fear, disgust, happiness, sadness and surprise. With the exception of the neutral expression, each of the six universal expressions includes four levels of intensity.

\subsubsection{Feature Subspace Dimensions}

The first experiment looks at the face recognition performance against feature subspace dimension. All 2,500 faces available in BU-3DFE database were used in the experiments. The faces were divided into ten subsets with each subset containing the same 100 subjects and all seven kinds of expressions. For each feature subspace dimension, one subset is selected for testing while the remaining subsets were used for training. Such experiments were repeated ten times with a different subset selected for testing each time. The faces in the training set are not used in testing. 
Table 1: Recognition comparison on BU-3DFE database.

\begin{tabular}{lcccccc}
\hline Expression & LPP-based & LDA-based & PCA-based & $\begin{array}{c}\text { Patch Geodesic } \\
\text { Moment }\end{array}$ & $\begin{array}{c}\text { Geodesic Polar } \\
\text { Representation }\end{array}$ & $\begin{array}{c}\text { Canonical Image } \\
\text { Representation }\end{array}$ \\
\hline Anger & $91 \%$ & $90 \%$ & $62 \%$ & $93 \%$ & - & - \\
Disgust & $92 \%$ & $85 \%$ & $53 \%$ & $79 \%$ & - & - \\
Fear & $91 \%$ & $89 \%$ & $51 \%$ & $82 \%$ & - & - \\
Happiness & $96 \%$ & $91 \%$ & $56 \%$ & $86 \%$ & - & - \\
Sadness & $88 \%$ & $93 \%$ & $61 \%$ & $85 \%$ & - & - \\
Surprise & $95 \%$ & $92 \%$ & $52 \%$ & $84 \%$ & - & - \\
\hline Overall & $\mathbf{9 2 . 1 \%}$ & $\mathbf{9 0 . 0} \%$ & $\mathbf{5 5 . 8 \%}$ & $\mathbf{8 4 . 8 \%}$ & $\mathbf{8 0 . 3 \%}$ & $\mathbf{7 7 . 2 \%}$ \\
\hline
\end{tabular}

Figure 4 shows the plots of rank-1 recognition rate versus the dimensions of the feature-subspace. It is seen that the LPP-based approach significantly outperforms PCA and is able to achieve similar results to LDA-based methods but with lower dimensional subspace used. The highest recognition rate for PCA is $79 \%$, whereas both LDA and LPP can reach $100 \%$ with 15 and 10 dimensional subspace used, respectively.

\subsubsection{Facial Expression}

The robustness to facial expression variation is an important aspect in the face recognition task. To see how the proposed methods cope with this issue, a comparison was made with that of the state-of-theart methods, including patch geodesic moments (Mpiperis, 2007), geodesic polar representation (Hajati, 2012) and canonical image representation (Bronstein, 2005). The same experimental protocol used in (Hajati, 2012) was applied in this work. The performance was measured in terms of rank-1 recognition accuracy and the Cumulative Matching Characteristics (CMC) (Rizvi, 1998). All the faces with the neutral expression were used for training, while the rest of database was used as the testing faces. The rank-1 recognition rates were tabulated in Table 1. From the table, it can be seen that among the proposed methods the LPP-based approach achieved the highest recognition rate with an average accuracy of $92 \%$, outperforming the other state-of-the-art expression-invariant techniques by around $8 \%$. It is worth noticing that its recognition rates for different expressions range from $88 \%$ to $96 \%$. This shows that the proposed scheme can handle facial expression changes well. The CMC of the proposed methods together with those benchmark methods are shown in Figure 5. From the figure, it can be noticed that the recognition rate of the LPP-based method is always higher.

\subsection{Experiments on Gavab Database}

The Gavab database is one of the most expressionrich and noise-prone 3-D face datasets available to the public (Moreno, 2004). It consists of 549 scans from 61 different subjects. The subjects, of which 45 are male and 16 are female, are all Caucasian. Each subject was scanned 9 times for different poses and expressions, giving six neutral scans and three scans with an expression. The scan with pose variations contains one scan while looking up $\left(+35^{\circ}\right)$, one while looking down $\left(-35^{\circ}\right)$, one for the left profile ($\left.90^{\circ}\right)$, one for the right profile $\left(+90^{\circ}\right)$ as well as one with random poses. The scans without pose changes include two different frontal facial scans, one with smile, and one with an accentuated laugh.

In order to evaluate the expression and pose invariance, the proposed approach is compared with the results achieved by the existing benchmark methods reported in (Drira, 2010). The same experimental protocol introduced in (Drira, 2010) was used here. The benchmark methods include sparse representation $(\mathrm{Li}, 2009)$, 3-D ridge images (Mahoor, 2009), concave and convex regions (Berretti, 2006) and elastic radial curves (Drira, 2010). In the experiment, one of the two frontal scans with the neutral expression provided for each person was taken as the training data. The rest of the scans were used for testing. Since the proposed approach is not designed for working on facial scans with large part of missing data, the scans for the left and right profiles were not included for testing. Table 2 illustrates the results of the rank-1 recognition accuracy for different categories of testing faces. From the table, it can be seen that the proposed approach provides a high recognition accuracy on both expression and pose variations, and outperforms majority of the existing methods with its performance very close to the best recognition accuracy achieved by the elastic radial curves (Drira, 2010). 
Table 2: Recognition comparison on Gavab database.

\begin{tabular}{lccccc}
\hline & LPP-based & $\begin{array}{c}\text { Sparse } \\
\text { Representation }\end{array}$ & $\begin{array}{c}\text { Ridge } \\
\text { Images }\end{array}$ & $\begin{array}{c}\text { Concave and } \\
\text { Convex Region }\end{array}$ & $\begin{array}{c}\text { Elastic } \\
\text { Radial Curves }\end{array}$ \\
\hline Neutral & $96 \%$ & $97 \%$ & $95 \%$ & $94 \%$ & $100 \%$ \\
Expressive & $91 \%$ & $83 \%$ & $72 \%$ & $81 \%$ & $95 \%$ \\
Neutral + Expressive & $93 \%$ & $95 \%$ & $78 \%$ & $84 \%$ & $95 \%$ \\
Looking up & $95 \%$ & - & $85 \%$ & $80 \%$ & $100 \%$ \\
Looking down & $94 \%$ & - & $87 \%$ & $79 \%$ & $98 \%$ \\
\hline
\end{tabular}

\section{CONCLUSIONS}

An effective 3-D shape matching scheme for pose and expression-invariant face recognition has been presented in this paper. The key contribution of the proposed work is to use isometric embedding shape representation and statistical modelling techniques to achieve accurate dense point correspondences and generate appropriate shapes for new 3-D face data. From the experimental results on the Gavab and BU3DFE database, it can be concluded that the LPPbased approach offers a recognition rate that can be as high as nearly $100 \%$ and is more expressioninvariant compared with the existing benchmark approaches. The research will be extended further by taking into consideration more practical factors. One possible extension for the work is to evaluate the ability of the proposed algorithm using more databases that are produced by different devices operated under various acquisition environments. The missing data problem can also be introduced and dealt with by modifying the shape matching scheme. Finally, more sophisticated pattern recognition methods can be applied to increase the overall performance of the proposed method.

\section{ACKNOWLEDGEMENTS}

The work presented in this paper was supported by the Engineering and Physical Sciences Research Council (Grant numbers EP/D077540/1 and EP/H024913/1) and the EU FP7 Project SEMEOTICONS.

\section{REFERENCES}

Berretti, S., Bimbo, A., Pala, P., 2006. 3D face recognition by modelling the arrangement of concave and convex regions. Adaptive Multimedia Retrieval, 108-118.
Bronstein, A., Bronstein, M., Kimmel, R., 2005. Threedimensional face recognition. International Journal of Computer Vision, 64(1), 5-30.

Bronstein, A., Bronstein, M., Kimmel, R., 2007. Expression-invariant representation of faces. IEEE Transactions on Image Processing, 16(1), 188-197.

Bronstein, M., Bronstein, A., Kimmel, R., Yavneh, I., (2006). Multigrid multidimensional scaling. Numerical Linear Algebra with Applications, 13, 149171.

Chang, K., Bowyer, K., Flynn, P., 2003. Multi-modal 2D and $3 \mathrm{D}$ biometrics for face recognition. Proceeding of IEEE Workshop Analysis and Modelling of Face and Gestures, 187-194.

Chang, K., Bowyer, K., Flynn, P., 2006. Multiple nose region matching for 3 -D face recognition under varying facial expression. IEEE Transactions on Pattern Analysis and Machine Intelligence, 28(10), 1695-1700.

Chellappa, R., Wilson, C., Sirohey, S., 1995. Human and machine recognition of faces: a survey. Proceeding of IEEE, 83(5), 705-740.

Chua, C., Han, F., Ho, Y., 2002. 3-D Human face recognition using point signature. Proceeding of International Conference Automatic Face and Gesture Recognition, 233-238.

Drira, H., Amor, B., Mohammed, D., Srivastava, A., 2010. Pose and expression-invariant 3-D face recognition using elastic radial curves. British Machine Vision Conference.

Elad, A., Kimmel, R., 2003. On bending invariant signatures for surfaces. IEEE Transactions on Pattern Analysis and Machine Intelligence, 25(10), 12851295.

Hastie, T., Tibshirani, R., Friedman, J., 2001. The elements of statistical learning. Springer Series in Statistic, Second Edition, Springer.

Hajati, F., Raie, A., Gao, Y., 2012. 2.5D face recognition using patch geodesic moments. Pattern Recognition, 45(2012), 969-982.

He, X., Yan, S., Hu, Y., Niyogi, P., Zhang, H., 2005. Face recognition using Laplacianfaces. IEEE Transactions on Pattern Analysis and Machine Intelligence. 27(3), 328-340.

Huang, D., Ardabilian, M., Wang, Y., Chen, L., 2012. 3-D face recognition using eLBP-based facial description and local feature hybrid matching. IEEE Transactions 
on Information Forensics and Security, 7(5), 15511565.

Kong, S., Heo, J., Abidi, B., Paik, J., Abidi, M., 2005. Recent advances in visual and infrared face recognition - a review. Computer Vision and Image Understanding, 97, 103-135.

Li, X., Jia, T., Zhang, H., 2009. Expression-insensitive 3D face recognition using sparse representation. Conference on Computer Vision and Pattern Recognition. 2575-2582.

Lu, Y., Zhou, J., Yu, S., 2003. A survey of face detection, extraction and recognition. Computing and Informatics Pattern Recognition, 22(2), 163-195.

Lu, X., Jain, A., 2008. Deformation modeling for robust 3D face matching. IEEE Transactions on Pattern Analysis and Machine Intelligence, 30(8), 1346-1356.

Mahoor, M., Abdel-Mottaleb, M., 2009. Face recognition based on 3D ridge images obtained from range data. Pattern Recognition. 42(3), 445-451.

Mohammadzade, H., Hatzinakos, D., 2013. Iterative closest normal point for $3 \mathrm{D}$ face recognition. IEEE Transaction on Pattern Analysis and machine intelligence. 35(2), 381-397.

Moreno, A., Sanchez, A., 2004. GavaDB: a 3D face database. COST Workshop on Biometrics on Internet: Fundamentals, Advances and Applications. 77-82.

Mpiperis, I., Malassiotis, S., Strintzis, M., 2007. 3-D face recognition with the geodesic polar representation. IEEE Transaction on Information Forensic and Security. 2(3), 537-547.

Pan, P., Wu, Z., Pan, Y., 2003. Automatic 3D face verification from range data. Proceeding of International Conference on Acoustic, Speech, and Signal Processing (ICASSP 03), 3, 193-196.

Jafri, R., Ardabilian, H., 2009. A survey of face recognition techniques. Journal of Information Processing Systems, 53(2), 41-66.

Jenkins, R., White, D., Van-Montfort, X., Burton, A., 2011. Variability in photos of the same face. Cognition, 121, 313-323.

Rizvi, S., Philips, P., Moon, H., 1998. The FERET verification test protocol for face recognition algorithms. IEEE International Conference on Automatic Face and Gesture Recognition.

Quan, W., Matuszewski, B., Shark, L., Ait-Boudaoud, D., 2009. Facial expression biometrics using statistical shape models. EURASIP Journal on Advances in Signal Processing.

Yin, L., Weim, X., Sun, Y., Wang, J., Rosato, M., 2006. A 3D facial expression database for facial behavior research. Proceeding of $7^{\text {th }}$ International Conference on Automatic Face and Gesture Recognition, 221-216. 